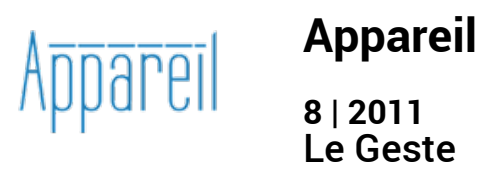

\title{
Du toucher au geste technique : la « technè des corps "
}

Patricia Ribault

\section{(2) OpenEdition}

1 Journals

Édition électronique

URL : http://journals.openedition.org/appareil/1315

DOI : 10.4000/appareil. 1315

ISSN : 2101-0714

Éditeur

MSH Paris Nord

Référence électronique

Patricia Ribault, «Du toucher au geste technique : la « technè des corps » », Appareil [En ligne], 8| 2011, mis en ligne le 02 novembre 2011, consulté le 30 juillet 2020. URL : http://journals.openedition.org/ appareil/1315; DOI : https://doi.org/10.4000/appareil.1315

Ce document a été généré automatiquement le 30 juillet 2020.

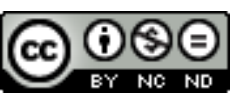

Appareil est mis à disposition selon les termes de la Licence Creative Commons Attribution - Pas d'Utilisation Commerciale - Pas de Modification 4.0 International. 


\title{
Du toucher au geste technique : la " technè des corps »
}

\author{
Patricia Ribault
}

\begin{abstract}
« On pourrait partir de là : un monde clos, fermé, plein, totalement immanent, un monde ou une chose, peu importe, qui serait si bien à soi et en soi qu'il ne se toucherait même pas et qu'on ne le toucherait pas non plus, un monde qui serait seul,

à soi et en soi, cela ne serait pas un corps ${ }^{1}$. »
\end{abstract}

1 Cela pourrait être ça, le monde d'avant la technique: un circuit fermé, une masse indifférenciée, comme le fond du monde, une chose en soi, lovée sur son fonctionnement biologique, se produisant et se reproduisant selon les mêmes lois naturelles immuables. Jean-Luc Nancy oppose ce monde-là à l'idée de corps, qui, lui est «l'ouvert », « l'extension », « l'exposition » au monde. Le monde devient corps par ce qui l'extériorise à lui-même. «Si le corps n'est pas masse, s'il n'est pas fermé sur soi et pénétré de soi, il est hors de soi. Il est l'être hors de soi ${ }^{2}$. " C'est le préfixe " ex » qui nous situe par rapport au reste du vivant. Depuis nos premiers gestes de transformation de la matière pour l'exposer à son devenir-forme, jusqu'aux objets techniques contemporains les plus sophistiqués, c'est dans le rapport de l'intérieur à l'extérieur que nous évoluons, sur un mode qui serait celui de l'existence, par opposition, ou plutôt par extension, à celui de la vie.

2 Selon J.-L. Nancy, le corps - de l'homme, du monde - s'expose à l'existence comme «techné des corps", système de création des corps connectés au monde par la technique. Dans quelle mesure peut-on dire que la technique «fait " le corps humain, fait corps avec l'humain, et par quels moyens? Doit-on filer la métaphore mécaniste d'un corps qui fonctionne à l'instar d'une machine, et, le cas échéant, en étudier les rouages et les mécanismes en tant qu'objet technique ? Faut-il au contraire considérer le corps comme l'élément déterminé par la nature qui inscrit l'homme dans sa condition d'être vivant? Ou bien le corps humain est-il un cas particulier qu'il faut penser selon une organologie qui lui est propre? Quel rapport entretient-il alors avec 
la technique qu'il produit? Tentons d'apporter quelques éléments de définition du geste, notamment du geste artisanal, dans son articulation entre le corps et la technique qu'il secrète, exsude hors de lui, mais aussi à partir de lui, donc par lui.

3 Nous envisagerons d'abord la technique comme moyen de pouvoir, au double sens auquel le mot nous renvoie : comme nom d'abord, c'est-à-dire comme moyen d'action et de domination, mais aussi comme verbe, qui pose la question de la technique en puissance: Que peut-elle? Nous verrons ensuite que le corps, pour produire de la technique, doit être technicisé lui-même, c'est-à-dire dressé, formé, ou dé-formé (toute la question est là). Nous analyserons les gestes techniques et leur impact sur l'intégrité voire l'identité corporelle de ceux qui s'y soumettent, avant d'aborder la question du toucher, qui, en tant que sens propre de l'exposition - ou de «l'expeausition » - ouvre l'espace d'un entre-deux, d'un partage des corps, qui est aussi celui d'une technicité. Nous distinguerons notamment la main qui touche des mains qui prennent et tenterons de définir le rapport propre à chacun de ces gestes.

\section{La technique comme pouvoir}

4 Au départ, la technique se pose toujours comme une hypothèse : «Et si on pouvait faire ceci ou cela avec ceci ou cela? ? «Et si... » disent les enfants, qui ne font rien d'autre que découvrir le monde des possibles. La technique, elle, découvre la forme dans l'informe, dé-couvre la puissance des ressources naturelles et, les découvrant, se découvre elle-même. Effet réflexif de la matière, qui, se donnant à l'œil, aux mains et à l'intelligence des hommes, les informent de ses potentiels.

5 À la manière de Bachelard, on peut imaginer que la glaise prise dans les mains, contenue, faisait déjà figure de contenant, induisant par ses qualités plastiques et métamorphiques sa forme à venir, à dé-couvrir. C'est la technicité des hommes qui révèle, qui réveille celle des éléments, a priori inépuisable. «Et si... » : début d'une idée, qui se forme en se formant, qui ré-fléchit en re-gardant ; aller-retour de la matière à l'imagination, en passant par le toucher. La réflexivité de la matière n'est pas uniquement visuelle, elle est aussi corporelle et s'inscrit dans les gestes de celui qui la manipule. Il ne s'agit pas d'une «intelligence de la main », comme on dit, mais plutôt d'une réceptivité, d'une disponibilité. «La main aussi a ses rêves, elle a ses hypothèses. Elle aide à connaître la matière dans son intimité. Elle aide donc à la rêver ${ }^{3}$. " Joie spéciale et primordiale du pétrissage des pâtes, «main dynamique », organe d'énergie avant d'être organe des formes, la main a ses raisons que la raison ignore.

6 «Et si on faisait... »: «Et si » est suivi d'un verbe à l'imparfait. Jamais un temps n'a si bien porté son nom ; l'imparfait ouvre à la perfectibilité, il est ce qui n'est pas achevé et est souvent suivi, selon la formule consacrée, d'un autre verbe au conditionnel : «...on pourrait... ». Oui mais, à condition de. On peut le dire autrement: l'imparfait serait le temps d'un manque. Pierre-Damien Huyghe analyse la technicité comme «le fait que l'homme est cet être qui touche à son monde. [...] L'homme est l'animal technicien ${ }^{4}$.» Toucher à son monde, cela signifie exister, donc "sortir du mode de l'être en subsistance ", et le moyen humain de pallier ce manque, c'est une émancipation par la technique, c'est-à-dire « toujours la mise en œuvre (en grec : l'én-ergie, en ergon) d'une puissance d'être ${ }^{5}$. »

7 L'« animal sans qualité » doit s'a-percevoir de son manque de technicité, c'est-à-dire sortir de sa condition strictement bio-logique (dictée par la nature), et y réagir. Cela 
implique un certain regard sur les choses, qui se distingue de la simple faculté de voir. La technique s'inscrit donc dans la matière comme un possible soumis à conditions. Celle de l'envisager d'abord. Elle doit se former dans l'esprit qui réfléchit, s'y représenter , s'y inventer. C'est ce que Gilbert Simondon qualifie d'« imagination technique, comme définie par une sensibilité particulière à la technicité des éléments ${ }^{6}$.» Ce n'est pas l'argile qui suggère le récipient, mais sa plasticité. Autrement dit, la technicité de l'argile est soumise à un ensemble d'éléments tels que sa consistance, la quantité d'eau qu'elle contient, sa résistance mécanique etc., qui induisent sa plasticité, et seulement alors devient-elle un élément susceptible de prendre forme dans le corps et l'esprit de celui qui la tourne et la retourne entre ses mains.

L'invention, qui est création de l'individu, suppose chez l'inventeur la connaissance intuitive de la technicité des éléments; l'invention s'accomplit à ce niveau intermédiaire entre le concret et l'abstrait qui est le niveau des schèmes, supposant l'existence préalable et la cohérence des représentations qui recouvrent la technicité de l'objet de symboles faisant partie d'une systématique et d'une dynamique imaginatives ${ }^{7}$.

8 « Entre le concret et l'abstrait », il y a l'intuition, cette qualité informe de l'esprit, entre le rationnel et l'irrationnel. Intueri en latin signifie «regarder attentivement». L'invention naîtrait de l'observation, de la concentration, du vouloir voir quelque chose, là où il n'y a encore rien. À moins, si l'on retient l'hypothèse de Gaston Bachelard, que ce ne soit pas la perception des images qui détermine le processus de l'imagination créatrice, et que «l'image perçue et l'image créée [soient] deux instances psychiques très différentes ». Les images imaginées seraient alors des «sublimations des archétypes plutôt que des reproductions de la réalité » et ces images sortiraient «du propre fonds humain ». S'attachant au caractère primitif de l'imagination créatrice, Bachelard se met en porte-à-faux de la philosophie réaliste et de la psychologie, selon lesquelles pour « richement combiner, il faut avoir beaucoup vu ».

9 À cette idée de "bien voir ", il préfère celle de "bien rêver, de rêver en restant fidèle à l'onirisme des archétypes qui sont enracinés dans l'inconscient humain ${ }^{8}$.» En d'autres termes, on peut supposer que les formes de la technique ne naissent pas forcément dans une " cohérence des représentations ", mais dans une " cohérence des rêveries de la matière ». La formule semble paradoxale, car comment des rêves, donc des vagabondages peuvent-ils être cohérents? Après tout, si délirants soient-ils, nos rêves révèlent aussi une logique cryptée, signe de la complexité imaginative de notre psychisme. L'hypothèse de Bachelard a le mérite d'ajouter une dimension expérimentale et tactile à l'intuition et à la représentation, qui évacuent la phase expérimentale de l'imagination matérielle, une imagination qui naît au contact de la matière, dans un tâtonnement approximatif et formateur.

D'une manière ou d'une autre, qu'il sera toujours difficile de déterminer avec certitude, l'organisation technique de la matière relève du rapport particulier de l'homme à son milieu. Les formes ne naissent pas d'une volonté déterminée ou d'une représentation précise, mais selon une dynamique, ou, pour reprendre un terme de Leroi-Gourhan, une tendance, qui aboutit aux faits techniques tels que nous les construisons.

La matière qui s'organise technomorphologiquement n'est pas passive, la tendance ne vient pas simplement d'une force organisatrice qui serait l'homme, elle n'appartient pas à une intention formatrice qui précéderait la fréquentation de la matière, et elle ne relève d'aucune maîtrise volontaire : elle s'opère, au fil du temps, par sélection de formes dans un rapport de l'être vivant humain à la matière qu'il 
organise et par laquelle il s'organise, où aucun des termes du rapport n'a le secret de l'autre'.

11 On retrouve dans cette synthèse de Bernard Stiegler l'idée d'échange, d'une organisation opérée et opérante, entre l'homme et son milieu, qui, par sa forme tout à fait singulière, à la fois zoologique et au-delà du zoologique, conduit l'homme à la prothéticité qui lui est propre et le fait sortir de sa condition strictement biologique. Il n'en demeure pas moins que ce rapport du "vivant humain » à la matière et les conditions de son apparition restent, au fond, un mystère.

12 La deuxième condition de possibilité de la technique serait celle de sa concrétisation. «Peut-on?». La matière résiste, ne se donne pas sans effort, le corps doit prendre sa mesure pour s'accorder avec elle et avec l'intention. Nous avons tendance à penser la technique comme un fait d'extériorisation par l'outil ; arrêtons-nous à présent sur ce que Marcel Mauss considère comme « une erreur fondamentale », car le corps est « le premier et le plus naturel objet technique, et en même temps moyen technique, de l'homme ${ }^{10}$ ». Considérons la phase de formation, les gestes techniques, voyons comment ils prennent forme dans le corps et à quel régime celui-ci doit se plier pour pouvoir agir et transformer le monde.

\section{Le corps dressé}

À partir de l'hypothèse selon laquelle "il n'existe peut-être pas de "façon naturelle" chez l'adulte ", Marcel Mauss dresse une liste désormais célèbre d'attitudes corporelles qu'on croyait innées ou personnelles, comme la manière de marcher, boire, donner naissance, faire l'amour, danser, nager, ou même dormir, qui relèvent en fait toutes de l'éducation, donc de la transmission, c'est-à-dire de la tradition. En cela elles sont des techniques, et parce qu'on peut dire qu'elles sont efficaces. Sans entrer dans le détail de cette énumération, on peut cependant mesurer l'étendue phénoménale du conditionnement dont nos moindres gestes font l'objet. Les sociétés imposent aux individus un usage déterminé de leur corps au profit d'une adaptation permanente de notre corps à l'usage. On peut, comme Marcel Mauss, parler de «dressage » :

Le dressage, comme le montage d'une machine, est la recherche, l'acquisition d'un rendement. Ici, c'est un rendement humain. Ces techniques sont donc les normes humaines du dressage humain. Ces procédés que nous appliquons aux animaux, les hommes se les sont volontairement appliqués à eux-mêmes et à leurs enfants ${ }^{11}$.

14 L'auteur introduit ici plusieurs notions fondamentales par rapport à l'idée de technique. D'abord, celle de "rendement humain». Elle fait écho avec celle du corps comme objet, puis moyen technique. En d'autres termes, le corps doit être technicisé pour produire de la technique, des objets techniques, mais aussi de l'identité socioculturelle. Le corps est le premier instrument dont se sert l'homme pour s'extérioriser. Cette éducation, ou ce dressage, assimilé au montage d'une machine, est peut-être aussi la première façon de se mettre « hors de lui » ou hors de la nature, si la nature consiste à suivre une évolution dictée par des lois naturelles de conservation et de reproduction. En mesurant ses gestes, l'être humain actualise sa maîtrise du monde, donc sa position d'extériorité et de domination par rapport à lui.

15 Gardons-nous cependant, des dérives de la philosophie mécaniste du xviI ${ }^{\mathrm{e}}$ siècle (Descartes en particulier) qui nous sont parvenues presque en l'état aujourd'hui. Ces théories assimilent le corps à une machine soumise au règne de l'esprit. Cette 
conception oublie que le corps n'est pas un automate qui répond à un programme : «le corps n'est pas nécessairement fluide, mais aussi bien dense, opaque, épais, non vide en tous les cas, de sorte qu'il fait opposition aux traversées d'informations à quoi les théories mécaniques voudraient l'assimiler ${ }^{12}$."

Pour Jean-Luc Nancy, les corps ne peuvent en aucun cas être réduits à des " objets techniques ${ }^{13}$ ». Ils sont des corps, «simplement» des corps, que le monde a créés comme tels, mais des corps intégrés dans un système d'appareillage: c'est l'« écotechnie» :

La "création" est la techné des corps. Notre monde crée le grand nombre des corps, il se crée en tant que monde des corps (mettant au jour ce qui toujours fut aussi sa vérité de monde). Notre monde est le monde de la "technique", le monde dont le cosmos, la nature, les dieux, le système complet dans sa jointure intime s'expose comme "technique": monde d'une écotechnie. L'écotechnie fonctionne avec des appareils techniques, sur lesquels elle nous branche de toutes parts. Mais ce qu'elle fait, ce sont nos corps, qu'elle met au monde et branche à ce système, nos corps qu'elle crée ainsi plus visibles, plus proliférants, plus polymorphes, plus pressés, plus en "masses" et "zones" que jamais ils ne furent. C'est dans la création des corps que l'écotechnie a ce sens qu'on lui cherche en vain dans les restes de ciel ou d'esprit ${ }^{14}$.

17 Il s'agit donc d'un «monde des corps » qui n'est ni immanent, ni transcendant, mais d'un monde de connexions. D'un monde qui n'existe pour nous - corps humains - qu'en tant que monde de la technique, c'est-à-dire de relations, d'appareillage, mais ce monde, cette écotechnie ne produit que des corps. La «techné des corps " fait des corps et fonctionne avec des appareils techniques. Un monde de corps articulés par la technique. C'est cette articulation ou cette " jointure intime ", donc, qu'il faudra penser comme contact et rupture à la fois. C'est bien le fait d'une articulation : elle joint deux membres tout en les séparant.

Revenons un instant à Marcel Mauss et à la notion de "norme humaine ". Le corps social érige des normes et contraint chaque individu, dès l'enfance, à se plier aux exigences d'un modèle, de la même façon qu'une conduite technique entraîne l'élaboration d'un corpus de gestes et d'attitudes normatives auxquelles l'individu doit se soumettre pour avoir prise sur le monde. La mise en place systématique de normes est l'un des principes de base de la technique, car tout en agissant, elle mesure, pèse, calcule, évalue et reproduit. Ceci est d'autant plus vrai aujourd'hui que les systèmes techniques sont mondialisés donc normalisés. Mais il ne faut pas confondre norme et normalisation. Au départ, il faut des instruments de mesure, des étalons, pour situer des modes opératoires. La norma, en latin, c'est l'équerre. Ou bien, selon Jean-Luc Nancy :

Pesée: création. Ce par quoi commence une création, sans pré-supposition de créateur. Sujet d'avant tout sujet, pesée, poussée exercée, reçue, communauté toute archi-primitive des forces [...] Les pesées distribuent l'étendue, extensions et intentions ${ }^{15}$.

19 Le monde, les corps, les objets se créent dans une balance où chacun pèse contre un autre, où l'un soupèse l'autre; le poids est une mesure qui fait norme, qui choisit et qui élimine. L'instrument technique est avant tout un repère visant à déterminer une exigence qui le dépasse et l'informe. On procède selon des normes conditionnées par l'exigence d'un résultat qui évolue lui-même en fonction de l'état des techniques et surtout, elles sont fixées par l'homme, en amont. "L'homme est un être normatif comme instaurateur de normes et comme opérateur se soumettant dans son opération 
à des normes » écrit Jean-Pierre Séris dans son ouvrage sur la technique ${ }^{16}$. Les modèles comme les normes imposent des règles qui, paradoxalement, doivent être suivies et ne demandent qu'à être transgressées, au nom du progrès - ou de l'évolution - pour instaurer de nouveaux modèles, qui, à leur tour, seront suivis, imités jusqu'à une nouvelle invention. En fin de compte, la norme projette, anticipe les modes d'usage et cours toujours en avant de son temps, à la recherche d'un avenir meilleur.

Le corps est le premier lieu de cette évaluation normative, qui aboutira ensuite à la sélection, la valorisation et l'élaboration des processus de fabrication. Visant la cohérence et le rendement, la normativité est inhérente à toute production. C'est selon une nécessaire adaptation que l'on fait usage du corps dans tout apprentissage technique, c'est-à-dire dans l'ensemble de gestes coordonnés et maîtrisés qui procèdent d'une méthode et parviennent à un but donné. C'est peut-être le seul dressage corporel dont nous soyons délibérément conscients. Les gestes du métier sont appris, intériorisés et employés selon le même processus que n'importe quelle autre « technique du corps ». Il n'y a de différence que dans la spécialisation et l'intensité de la formation. Le corps lui-même devient matière à former, dans un même mouvement réflexif qui va de la matière au corps et du corps à la matière. Le pouvoir, ici, est celui que donne la «formule » à appliquer au corps pour parvenir à ses fins, à ce résultat tangible, cet objet que l'on a produit, cette partition que l'on a déchiffrée, cette vitesse que l'on a atteinte.

21 Source de tellement d'efforts et de gratifications à la fois, le modelage corporel est une voie dans laquelle l'être s'engage tout entier, car il entraîne une modification irréversible de la perception, donc de l'être au monde. Chaque spécialiste le sait : on ne perçoit plus le monde de la même manière après avoir incorporé une technique. Le photographe pose un regard "cadré » sur le monde, le musicien tend l'oreille, le cuisinier est sensible aux odeurs et aux goûts, l'artisan au toucher et à tout ce qui touche (justement) à la matière qu'il travaille. L'homme modèle donc son corps, le forme - et/ou le déforme - selon ses besoins ou ses pratiques, qu'elles soient techniques ou artistiques.

La question qui se pose maintenant est celle de ses limites : peut-on dire que le corps soumis à la technique est un corps arraché à la nature, " dénaturé17 " et si tel est le cas, où se situe la limite entre exercice et déformation du corps?

\section{Le geste technique}

Un geste technique est un geste nécessairement maîtrisé. Faire de son propre corps un moyen technique appareillé par des objets techniques (outils et/ou machines) revient à imprimer, à graver au plus profond de soi une technicité qui devient aussi une identité. On parle souvent de "déformation professionnelle"; mais une déformation par rapport à quoi ? À un état de neutralité, de «naturalité » du corps? Tout corps se déforme, par nature, avec l'âge, si l'on admet que la forme d'un corps est celle qui correspond à la pleine possession de ses moyens. La déformation est donc une dégradation propre au vieillissement. Un corps vieillissant n'est pas un corps déformé, c'est un corps qui suit la logique de dégradation de toute chose vivante. Un corps déformé perd une partie de son unité en tant que forme et fonction, parce qu'il a subi, dans une certaine mesure, une violence. Violence d'un choc accidentel, ou violence d'un conditionnement « contre-nature ». 
24 Mais l'apprentissage d'une technique est-il une forme de violence portée au corps humain? Un corps dont on a vu qu'il est essentiellement indéterminé jusqu'à ce qu'il commence à sécréter des outils, donc des spécialisations techniques sans lesquelles on ne peut pourtant l'envisager. Ce corps-là, qui devient humain par les prothèses artificielles qu'il apprend à produire en adaptant ses gestes à la matière. Un corps donc, qui est humain parce qu'il peut se maîtriser, se con-former au monde qu'il transforme. Un corps enfin dont le propre est de sortir des strictes limites que lui a données la nature.

Autrement dit, où s'arrête la formation et où commence la violence?

Dès le départ, la situation est contradictoire. D'un côté, on a un devenir humain qui passe par des gestes perfectibles qui nous amènent à transformer le monde et à produire des outils, des prothèses, des objets. De l'autre, notre appareil corporel tend à rester neutre, lisse, indéterminé et notre humanité se cherche dans le développement de ses «manières» et de sa "connaissance». Nous mettons alors toute notre ingéniosité à artificialiser notre technicité (ce qui est de plus en plus notre cas) et à exister par ailleurs.

C'est tout le paradoxe de notre ascension technique : nous avons d'abord déployé des trésors d'habileté, d'ingéniosité et de force pour nous affranchir des lois naturelles, transformer le monde et le soumettre à nos besoins, mais nous avons tant et si bien fait que nous sommes presque parvenus à retrouver notre indétermination physique première, entourés que nous sommes à présent d'objets réalisés par des ensembles techniques mécanisés dont le fonctionnement ne nous a jamais été si étranger. D'où la nécessité, selon G. Simondon, d'étudier l'évolution des objets techniques dans une perspective qui les rapproche de la réalité humaine dont ils sont issus, ou celle, selon Bruno Latour, d'approfondir les relations réciproques entre les "humains" et les "non-humains», entre les différents agents de la médiation technique. B. Latour démontre en effet que les artefacts sont autant façonnés par nous qu'ils nous façonnent et qu'il faut «apprendre à attribuer - à redistribuer - les actions à beaucoup plus d'agents que ne le prévoient les scénarios matérialiste ou sociologique ${ }^{18}$.»

Cela revient à dire qu'il existe un mouvement de va-et-vient, un échange complexe et sinueux entre les techniques et nous, qui modifie à chaque moment de son évolution les termes de notre rapport au monde. Ainsi, nos artefacts ne sont pas posés là, en-dehors de nous comme des choses extérieures et objectivables, comme des intermédiaires, « ils méritent d'être accueillis dans notre culture intellectuelle en tant qu'acteurs sociaux à part entière. Médiatisent-ils nos actions? Non, ils sont nous ${ }^{19}$." D'où l'intérêt d'approfondir les modes opératoires des gestes techniques.

Dans l'article intitulé "Les coordonnées culturelles du geste ${ }^{20}$ ", la première remarque d'Éric Gallais et Ali Ahmed Saïd reprend la distinction classique et dévalorisante entre l'activité manuelle et celle d'ordre intellectuel, pour la réduire à une ineptie quand il s'agit du geste technique: il n'y pas de geste qui soit purement manuel sans une relation de complémentarité avec un travail intellectuel. "L'indissociable complémentarité des activités manuelles et intellectuelles s'observe à tous les niveaux de la réalisation d'un projet ou d'un chantier ${ }^{21}$. " Et de donner l'exemple de tous les problèmes qu'il faut résoudre pour régler les dysfonctionnements éventuels d'un outil. Parce qu'il doit « gérer l'ensemble des tenants et des aboutissants de son travail, ce qui nécessite la mise en œuvre de compétences multiples, dans un cadre souvent 
complexe ${ }^{22}$ ", l'artisan doit donc constamment chercher des solutions inventives et efficaces aux problèmes qui se posent à lui.

On retrouve cette attitude dans le rapport - souvent paradoxal - des artisans à l'innovation: dans leur pratique quotidienne, ils recherchent sans cesse de nouveaux matériaux ou de nouveaux coups de main, gestes, ou astuces qui leur faciliteront la tâche. Pourtant, dès qu'il s'agit d'introduire une innovation « un peu trop nouvelle » ou théorique dans leur travail, ils se montrent souvent réfractaires, car l'habileté naît de l'habitude et de la répétition, créant un équilibre que la nouveauté met en danger. Ainsi, selon Michel Guérin, "l'artisan est technologiquement conservateur, car l'habitude-habileté réprime les innovations de l'intelligence symbolique et s'efforce de maintenir le plus possible dans le "moteur" ce qui pourrait être traité et exploité au niveau central ${ }^{23}$. " L'artisan est donc souvent tiraillé entre la perfectibilité de son geste et sa curiosité professionnelle, qui doit le mener à explorer de nouveaux territoires techniques et formels.

31 C'est d'ailleurs ce qu'il « vise » qui fera la différence entre le geste technique et le geste artistique. L'outil et la machine, dans un cas comme dans l'autre, font partie du geste, et visent à la réalisation d'une œuvre, mais c'est la façon de faire et la finalité qui changent. Le geste technique vise la maîtrise (sans pour autant exclure les errements et les tâtonnements) alors que le geste artistique cherche l'œuvre tout en la faisant. Il est moyen d'expression voire fin en soi. Toutefois, à l'origine, l'un est indissociable de l'autre :

Parce que le geste indique une liaison forte à la vie tout en esquissant les mondes technique et esthétique, nous nous persuadons de l'artifice qu'il y aurait à séparer brutalement le technique, l'affectif et le symbolique ${ }^{24}$.

Entendu d'abord comme invention, trouvaille du corps, donc expression, il devient ensuite habitude, maitrise et tradition, donc transmission et imitation. Ce qui le rend technique est donc la notion d'efficacité, le pouvoir de relation et de transformation qu'il établit entre l'homme et son milieu. Il se pose comme un intermédiaire entre le corps - la main notamment - et l'esprit, entre la matière et le corps, entre la nature et la culture : ouverture et perception d'abord, contact et connaissance ensuite, habileté et habitude enfin. Mais avant d'être traité comme information, le geste est d'abord une découverte "sauvage ", une émotion, une communication directe et sensuelle entre l'intérieur et l'extérieur, la matière et la chair. La percussion est née de la rencontre d'une main et d'une pierre, mais aussi d'une agressivité et d'une impuissance, avant de devenir un geste technique.

33 Sensible avant tout, comme une pellicule photographique à la lumière, le corps est donc le premier instrument de mesure, d'écoute, d'action; il reçoit les pressions, les impressions, la consistance des matières auxquelles il donne corps. Tour à tour maître et valet, formé et formateur, il est ouvert.

Avec le corps, nous parlons de ce qui est ouvert et infini, de ce qui est l'ouvert de la clôture même, l'infini du fini lui-même. C'est cela que je veux essayer de développer : le corps, c'est l'ouvert. Et pour qu'il y ait ouverture, il faut qu'il y ait quelque chose de fermé, il faut que l'on touche à la fermeture. Toucher à ce qui est fermé, c'est déjà ouvrir. Peut-être qu'il n'y a jamais d'ouverture que par un toucher ou par une touche. Et ouvrir - toucher - ce n'est pas déchirer, démembrer, détruire ${ }^{25}$.

34 Toucher, c'est donc ouvrir quelque chose de fermé, ou d'inerte, comme peut l'être la matière à l'état brut. Par le toucher, qui est connaissance d'abord et transformation 
ensuite, le geste déploie les qualités ou la technicité contenues dans la matière et en lui-même. C'est sous cet angle que nous aborderons à présent le toucher, comme surface de connexion d'une part, mais aussi comme risque.

\section{Le toucher}

Le toucher est le seul sens par lequel notre rapport au monde nous échappe. Un rapport qui ne passe pas par un délai entre le sujet pensant et l'objet, mais qui, dans la simultanéité de la sensation, s'approche au plus près du monde au point de s'y mêler et s'y confondre. Le sujet touchant-touché est directement saisi par ce monde dont il prend d'ordinaire la mesure par l'intermédiaire de ses autres sens et de sa raison, qui mettent à distance sa relation aux choses. Or, comme on va bientôt le voir, cette simultanéité n'est que temporelle et il s'agit de ne pas la confondre avec une immédiateté qui n'est pas le propre du toucher.

En outre, on ne peut faire abstraction de ce que l'on touche. Le toucher est le sens de la présence. Commun à tous les animaux, il est aussi celui qui nous distingue le plus d'eux, par l'usage que l'on en fait, dans la subtilité de ses nuances. Il est le paradigme du sensible, du sentir, à la fois universel et profondément humain en tant qu'émotion. Il est le plus corporel de tous nos sens, car le corps entier est sentant, même si la main est souvent l'interlocutrice privilégiée du toucher. C'est par le toucher que le monde s'est ouvert et détaché de nous, instituant dans cet abîme vertigineux de la technique un toucher curieux, différencié, observateur, manipulateur et surtout, surtout, producteur.

Pour Merleau-Ponty, chaque sens se différencie des autres par sa propre perception des objets.

Chaque organe des sens interroge l'objet à sa manière, il est l'agent d'un certain type de synthèse, mais [...] on ne peut pas refuser au toucher la spatialité au sens de saisie des coexistences. Le fait même que la vision véritable se prépare au cours d'une phase de transition et par une sorte de toucher avec les yeux ne se comprendrait pas s'il n'y avait un champ tactile quasi-spatial, où les premières perceptions visuelles puissent s'insérer ${ }^{26}$.

Le toucher comme "saisie des coexistences ». Cette magnifique formule pourrait aussi s'appliquer au mode de perception des artisans, qui, bien qu'ils ne soient pas aveugles, accordent au toucher une valeur essentielle, eux qui ont développé leur appréhension du monde et de l'espace par le toucher. La nécessité, pour un artisan, de «saisir les coexistences » de la matière et des formes, passe nécessairement par le toucher. La vision, en effet, correspond à un être au monde d'observateur, de spectateur d'un espace distancié, dans sa globalité ou ses particularités, mais d'un espace auquel on ne touche pas. Ou auquel on ne touche pas encore. La spacio-temporalité du toucher est tout autre. Là où la vue embrasse l'ensemble dans son champ de vision, la surface d'exposition d'un corps touchant-touché est localisée à la partie en contact. Ce contact se distingue aussi par une sensation de l'être, qui, simultanément, sent et est senti.

Mais ce n'est pas tout. Le toucher n'a pas seulement pour objet le tangible, mais aussi le non-tangible: comment toucher à l'intouchable? Limites du corps et de l'âme qui cohabitent tant bien que mal sous le même toit, dissociés à l'infini par la philosophie et pourtant indissociables dans leur relation. Le toucher serait-il donc le sens qui en finit avec le dualisme âme/corps? 
C'est la thèse que défend J.-L. Nancy dans De l'âme, lorsqu'il il parle du corps, mais du corps révélé par l'âme: "l'âme est un nom pour l'expérience que le corps $e s t^{27}$. ». Il démontre que pour Aristote, « corps veut dire très exactement l'âme qui se sent corps. Ou : l'âme est le nom du sentir du corps [...] le dedans qui se sent dehors ${ }^{28}$ ». Avant d'ajouter que "le "soi-corps" ou "l'être à soi du corps" c'est le "Sujet", le "Je" et que "je" est une touche."

«Je » n'est rien d'autre que la singularité d'une touche, d'une touche en tant qu'une touche est toujours à la fois active et passive et qu'une touche évoque quelque chose de ponctuel - une touche au sens d'une touche de couleur, au sens de la touche d'un pianiste, et pourquoi pas au sens où on disait dans le vieil argot, faire une touche. L'unité d'un corps, sa singularité, c'est l'unité d'une touche, de toutes les touches (de tous les touchers) de ce corps. Et c'est cette unité qui peut faire un moi, une identitéé ${ }^{2}$.

41 Une touche, qu'on imagine posée comme ça, du bout des doigts, comme la marque d'une identité, d'une façon propre à chacun, à la différence des techniques du corps dont parlait Marcel Mauss. La touche, légère ou appuyée, c'est la façon de toucher du peintre, du pianiste, de l'artisan, de tout un chacun enfin, c'est le moment où l'on passe de l'intérieur à l'extérieur, où l'on touche à soi-même, où l'« on se sent comme un dehors.» Rapport de l'âme au corps qui ne peut donc se penser qu'en tant qu'expérience du corps, non dans son intériorité mais au contraire dans un rapport d'ex-position, d'ex-tériorité, un «se sentir » qui fait ex-ister l'âme dans le corps. Le corps lui-même est l'organe $d u$ toucher, l'expérience de toucher et d'être touché. Il est nécessairement ouvert.

Le corps est l'expérience de toucher indéfiniment à l'intouchable, mais au sens où l'intouchable n'est rien qui soit derrière, ni un intérieur ou un dedans, ni une masse, ni un Dieu. L'intouchable, c'est que ça touche. On peut aussi employer un autre mot pour dire cela : ce qui touche, ce par quoi on est touché, c'est de l'ordre de l'émotion. Émotion est un mot très affaibli pour nous, mais émotion, cela veut dire : mis en mouvement, mis en branle, ébranlé, affecté, entamé ${ }^{30}$.

42 À lire ces lignes, on comprend qu'être touché c'est d'abord l'être dans son corps, quand bien même il s'agirait d'un état d'âme. Le toucher relève de l'émotion, qui porte en elle la dynamique du mouvement. Fondamentalement, la relation à la matière est donc un lien émotionnel.

C'est à partir de l'idée de mouvement qu'on a pu fonder celle d'une technique née dans la matière comme source de réflexion et de gestes techniques fondateurs; à partir de l'émotion que se fonde l'imagination matérielle développée par Bachelard. Elle saisit celui qui l'éprouve, elle le fait ré-agir, c'est-à-dire agir en réponse à une stimulation, elle bouscule l'ordre établi. On peut le dire autrement : elle dessaisit ce qui était stable, provoque l'Autre au cœur de sa corporéité. Elle se manifeste d'abord par des réactions physiques: on rougit, on s'échauffe, on tremble. À vrai dire elle est d'abord et exclusivement un mouvement du corps, justement parce que l'âme se trouve dessaisie de ce qu'elle connaissait. Elle est l'épreuve d'un désordre et d'un déséquilibre.

En fin de compte, on peut imaginer que les premiers gestes techniques sont nés non pas d'une pensée réfléchie ou d'une décision de la volonté mais plutôt d'émotions réfléchies dans la matière. Le toucher à ce moment-là devient miroir, vecteur, topos, là où ça $\mathrm{a}$ lieu, là où le "ça » de la technique s'ébauche dans un toucher qui n'est plus seulement toucher, mais déjà geste, mise en forme. Le toucher, au point de son évolution en geste, donc en mouvement, n'est plus dans l'immédiateté, il se dépose, comme le lit d'un 
fleuve, dans la matière, tout en s'imprimant dans l'esprit. Le toucher relève de la sensibilité d'une pellicule photographique sur laquelle s'enregistre l'image du monde, au moment où l'on déclenche un mécanisme.

Comme il n'y a pas de toucher sans intervalle (pellicule, membrane "séparant la peau des choses et que nous ne sentons pas"), et comme cette interposition se dérobe, eh bien, l'oubli de cette médiation produit la croyance illusoire à

l'immédiateté du contact ${ }^{31}$. greffe prenne ${ }^{33}$ ", Catherine Vincent analyse les conséquences psychologiques des greffes d'organes. Elle s'interroge ainsi par rapport à ceux qui reçoivent les parties visibles du corps comme Denis Chatelier, "premier homme au monde à avoir reçu en janvier 2000 [...] les avant-bras et les mains d'un autre. Des mains auxquelles il fallut réapprendre le mouvement, le toucher, la préhension. Et les souvenirs. » Le siège de la mémoire n'est pas seulement dans le cerveau, mais aussi dans notre corps :

Lorsque notre main touche un objet, ce geste est suivi de l'enregistrement de signes dans notre cortex, auxquels correspondront des formes, puis des souvenirs qui seront réactivés lorsque notre main, à nouveau, touchera cet objet. Mais lorsqu'on reçoit une nouvelle main, de quoi va-t-elle se souvenir $?^{34}$

L'article ne nous dit pas si ces «nouvelles » mains avaient des souvenirs propres, mais on apprend qu'au bout de trois ans, «cet ancien peintre en bâtiment, qui a repris une activité professionnelle depuis 2003, avait progressivement retrouvé une sensibilité jusqu'au bout des doigts. Et que son cerveau, dans le même temps, avait transféré aux nouveaux membres les représentations mentales des anciens.» Prouesse de la 
médecine et de la nature qui force l'admiration et l'imagination. Mais si le cerveau dirige la sensation, le toucher, qu'en est-il des gestes techniques? Cet homme est peintre en bâtiment, il a donc un certain savoir-faire, qu'est-il devenu? Il ne suffit pas de transférer des souvenirs, mais une mesure, une délicatesse, une force, toute une gestualité qui se forme aussi à partir de sa propre morphologie. Jusqu'où peut-on s'approprier le toucher d'un autre, le corps d'un autre lorsque l'expérience même de son corps est celle d'un technicien, un porteur d'outils?

Jean-Luc Nancy, qui a reçu le cœur d'un ou d'une autre, écrit dans L'Intrus : «Accueillir l'étranger, il faut bien que ce soit aussi éprouver son intrusion [...] Mais l'étranger insiste et fait intrusion. C'est cela qui n'est pas facile à recevoir, ni peut-être à concevoir....35 » Quelle perception a-t-on de son propre corps lorsque, touché au cœur, au cœur même de l'organe du sentir (si l'on en croit Aristote), il devient le théâtre d'une altérité qui le rend étranger à soi-même? Que penser des "progrès » des sciences et techniques lorsque, comme J. L. Nancy, on en vient à penser que :

L'intrus n'est pas un autre que moi-même et l'homme lui-même. Pas un autre que le même qui n'en finit pas de s'altérer, à la fois aiguisé et épuisé, dénudé et suréquipé, intrus dans le monde aussi bien qu'en soi-même, inquiétante poussée de l'étrange, conatus d'une infinité excroissante ${ }^{36}$.

51 Est-ce la finalité de la technicité de l'homme, de tant et si bien s'extérioriser qu'il finit par se rendre étranger au monde et à lui-même, ou bien l'homme est-il hors de lui par définition? Est-il en train de devenir ce qu'il est : «le plus terrifiant et le plus troublant technicien [...] qui dénature et refait la nature, qui recrée la création, qui la ressort de rien et qui, peut-être, la reconduit à rien ${ }^{37}$ "? La technique relaie de plus en plus l'organique, corrige le vivant, repousse l'arrivée de la mort dans l'organisme qu'elle morcelle, dissocie dans toutes ses possibilités combinatoires, hésitant entre la prothèse, l'orthèse et la greffe, cherchant par tous les moyens à augmenter notre « rendement " corporel. La prothèse relève de la performance et «fonctionne » conjointement avec l'homme. Le corps semble rejoindre la machine au point de son fonctionnement, de sa mécanique, de sa causalité : "ça marche», dit-on quand l'appareil répond aux commandes.

52 Malgré le scepticisme des uns et des autres et quand bien même ils auraient, comme Jean-Luc Nancy, subi (ou plutôt enduré) des interventions si lourdes que leur vie, après, semble soumise à un "régime permanent de l'intrusion» comme il le dit lui-même, cette « écotechnie » qui « branche les corps de toutes parts »- et ici au sens propre du terme - ne tend-elle pas malgré tout au mieux-être de l'homme? Non pas seulement au mieux-vivre biologique, mais au mieux-exister, au mieux-être-au-monde? Ou tout simplement à « rester au monde », quelles qu'en soient les conséquences?

Ne serait-ce que pour écrire ce livre bouleversant, L'Intrus, et tous ceux qui ont suivi, la peine de J.-L. Nancy ne valait-elle pas - pour nous du moins - la peine d'être vécue ? C'est bien entendu la lectrice que je suis qui parle, et le philosophe, l'homme en souffrance n'est peut-être pas de cet avis. Toujours est-il que ses livres, dans lesquels se déposent forcément les traces philosophiques de ses épreuves (je pense à Corpus), offrent au monde une vision du corps sans précédent dans l'histoire de la philosophie. Et c'est ce qui fera écrire à Derrida les trois cent cinquante pages de Le toucher, Jean-Luc Nancy et en particulier ceci :

C'est entre 1985 et 1991 [...] que le corpus est, plus que touché, sommé, presque violé, pénétré, dominé par l'opération qui vient inscrire du « toucher » au cœur de toute l'écriture. [...] L'opération dont je parle est donc contemporaine d'une 
opération " chirurgicale ", comme on dit, faite à la main, la main de l'autre, et donc au toucher, même si, comme toujours, la machine et la technologie y furent indispensables (et dans tous les chapitres suivants, c'est la pensée d'une techné des corps comme pensée du supplément prothétique qui fera, me semble-t-il, la différence la plus marquée entre le discours de Nancy et d'autres discours, plus ou moins contemporains, sur le « corps propre » ou la « chair ») $)^{38}$.

Intrusion, donc, dans la vie du philosophe (à vrai dire dans la vie des deux philosophes), donc dans son ceuvre, d'une "opération" qui, en tant que toucher, se reflète, se confond, dans l'existence et la pensée de celui qui en fait l'épreuve. Étymologie commune de l'œuvre intellectuelle de l'un et de l'operare manuel de l'autre (toujours absent d'ailleurs), qui se retrouvent dans l'entre-deux du toucher à. À ce corps d'une part, et à l'âme d'autre part, qui nous donnent la preuve par l'œuvre qu'ils ne font qu'un. « Techné des corps » qui prouve encore une fois son irréductibilité à la manœuvre technique et à la pensée mécaniste du corps. Précisément car c'est le corps qui accueille la technique, celui de l'homme qui se cherche, qui cherche à se toucher - lui-même, l'un l'autre, les uns les autres -, qui s'extériorise par cette « techné ».

Ainsi, le toucher du corps sentant, ouvert à ce qui fait événement est le lieu du devenir technique. Dans cet espacement entre le touchant et le touché, s'inscrit la technicité de l'homme, son " étrangeté » à lui-même ou son extériorité, qui n'est rien d'autre qu'une relation de complémentarité, ou, comme l'analyse Derrida, de « supplémentarité » :

Une technique, une technique machinale (depuis toujours mais combien plus manifestement désormais dans les machines présentes et à venir), c'est ce qui peut discriminer ou " percevoir » en fonctionnant au simulacre du « sensible » [...] mais qui ne " sent " pas, ne se rend pas à la distribution ou à la hiérarchisation des "cinq sens" et surtout ne se sent pas sentir - et reste donc, là même ou une machine supplée le sens, anesthétique. Et là où manque par exemple le rapport à soi d'un "se toucher ", fût-ce dans ce que nous appelons notre " corps propre ", la place est ouverte pour de la machine, de la prothèse, du substitut métonymique et du "un sens pour un autre ${ }^{39}$.

Retour à l'ordre des choses : ce n'est donc pas le corps qui est une machine mais bien la machine qui fonctionne comme le sensible, mais un sensible qui ne coordonne pas les sensations, qui n'opère pas de synthèse des impressions sensibles et surtout qui « ne se sent pas sentir ». Un corps, donc, irréductible à une pensée mécaniste. La machine, la prothèse ou l'outil s'inscrivent là où manque le contact, dans l'écart, toujours lié chez Nancy avec le toucher. Ils produisent du contact, des connexions, de l'avoir-lieu: "quelque part se distribue sur de très longs circuits techniques, "quelque part" est la technique, notre contact discret, puissant, disséminé ${ }^{40} »$.

On ne peut toutefois aborder la question du toucher sans aborder les vecteurs de cette conquête de la technique que sont les mains. Au moment où l'on passera de la main qui touche à celle qui prend, on pourra, sans risque de confusion, passer du singulier au pluriel, car là où la main est instance du toucher, de l'exploration, les mains sont celles qui opèrent ce passage de la sensation au travail. Rares sont les artisans manchots.

\section{La main qui touche}

Selon Michel Serres, les mains de l'homme "ne sont propres à rien mais bonnes à tout. » Des mains qui, avant de saisir, tenir, manipuler, frapper, arracher, touchent et, 
touchant, sont touchées. Dans leur rapport à la matière, c'est cette réciprocité qui nous intéresse. l'éviter. Trop évidente, réductrice. Le toucher ne s'arrête pas à la main exploratrice, il est bien plus, il est l'être au monde, l'ensemble du sentir, l'espacement d'avant la distinction ou la confusion. Toutefois, Derrida revient, lui, sur cette écartement de la main et des doigts, cherchant chez d'autres penseurs, Maine de Biran et Merleau-Ponty notamment, ce qui ferait le propre de notre main d'«animal pensant». Le toucher manuel est, dit-il, associé chez de Biran à la résistance puis à l'effort: "tout se passe comme si tout passait par la main, par la main de l'homme, par les trois temps de la main de l'homme ${ }^{44}$.» Trois temps que l'on pourrait résumer ainsi: d'abord la "sensation pure et purement passive ", la réceptivité de la main touchée (disons, pour reprendre un terme de Nancy, l'émotion). Puis l'évaluation, l'analyse de cette sensation (l'information est parvenue au cerveau, la matière circule de la main au cortex par voie nerveuse, le sujet interprète la sensation). Enfin la décision, le jugement rendu par le sujet, qui fera ré-agir, ré-pondre d'une manière ou d'une autre à cette sensation. C'est ce que Biran appelle "l'effort», pris entre un réflexe (celui de refermer la main) et un jugement, qui en décidera peut-être autrement: "Sans un sujet ou une volonté qui détermine le mouvement, sans un terme qui résiste, il n'y a point d'effort, et sans effort point de connaissance, point de perception d'aucune espèce ${ }^{45}$. »

62 La main ici n'est pas seulement une partie du corps réceptive comme une autre, elle est aussi active, ou plutôt réactive : aller-retour d'une impression suivie d'un geste qui répond à l'analyse du sujet sentant, du sujet pensant. Bien sûr, elle est l'organe par excellence d'une réponse "articulée», puisque, grâce aux doigts, elle est particulièrement sensible, mobile, habile. Aucune autre partie de notre corps ne peut réagir à la sensation de façon si active. Voilà pourquoi la main fait - naturellement figure d'instrument d'analyse. Mais en fin de compte, ce n'est pas la main qui analyse, c'est le cerveau, et s'il n'analyse pas, on reste alors dans la perception pure, dans 
l'émotion, la réaction corporelle non pilotée par l'organe de classement et de commande qu'est le cerveau, donc la pensée, l'effort ou la volonté.

Jacques Derrida, non sans ironie, qualifie le rapport qu'entretient de Biran avec la main d'« humainisme", qui "implique la même hiérarchie téléologique, et les mêmes présupposés, quant à l'animal; il traduit le même savoir, le même vouloir, la même volonté de savoir, mais souvent aussi le même obscurantisme ${ }^{46}$. » Il faut en effet insister sur le caractère arbitraire d'une main qui serait « connaissante ». Plus que toute autre partie du corps (sauf, peut-être, les lèvres) elle est sensible au toucher, réceptive et réagissante, mais on ne peut confondre le tact, le toucher, et l'organe qui le représente peut-être le mieux, la main ${ }^{47}$. C'est parce qu'elle est l'organe agissant de la volonté ou de l'émotion, voire de l'imagination, et parce qu'elle permet la réalisation de nos œuvres, qu'elle mérite ses lettres de noblesse; non parce qu'elle serait, elle-même, « intelligente ». On reconnaîtra le privilège de l'organe tel qu'il est constitué, de chair, d'os, de muscles, de doigts et d'articulations ; bref, on reconnaîtra sa forme, mais c'est le sujet qui est habile et c'est sa dextérité d'homme doué que l'on reconnaîtra, et non celle de sa main, comme si elle était détachée de lui.

De même que le corps n'est pas une machine mais un organisme, la main n'est ni un instrument, ni un outil, mais un organe spécialisé (ou, en l'occurrence, peu spécialisé) rattaché à l'ensemble des organes qu'est l'organisme. On sent bien que le corps relève d'une organisation qui tend à l'instrumentalisation de ses parties (l'organon grec est d'abord un instrument de travail, de musique, avant d'être un "élément [du corps] remplissant une fonction »), mais il est une structure opérante et structurée qui se forme selon une répartition des énergies (on retrouve la force de travail, ergon, à l'œuvre) et un assemblage des différentes parties entre elles.

La main en tant qu'organe, n'est pas soumise au cerveau, elle remplit une ou plusieurs fonction(s), au même titre que les reins, les genoux ou la langue. En revanche, son activité, ses gestes et sa mémoire dépendent bien d'un siège nerveux situé dans le cortex, qui envoie des ordres, que la main exécutera plus ou moins bien, car dans son épaisseur charnelle, elle résiste à la pensée, ou du moins, elle l'interprète. Il n'existe pas de perfection dans la manualité, ce qui donne d'ailleurs à l'artisanat d'aujourd'hui (donc à l'artisanat au temps de la production mécanisée) un attrait qu'il n'a jamais connu avant l'ère de l'industrie : le charme d'une trace ou d'une présence manifestée par la tactilité qu'il porte en lui, quand bien même cette manualité se présenterait comme un défaut, ou une imperfection. Car c'est bien de cela qu'il s'agit: la production industrielle se présente comme l'exécution parfaite et reproductible à l'infini d'un projet, alors que la production artisanale reste une interprétation par l'artisan.

Le toucher fait toujours l'expérience en premier lieu de l'espace, de l'altérité, de l'inconnu, et son épaisseur charnelle, "résistante " au réflexe ou à la commande, est justement ce qui ne fait pas de la main «l'esclave servile» du cerveau dont parlait Baudelaire. La main qui touche interroge la matière avant d'y répondre. Sensible avant d'être active, elle fait l'épreuve de l'extériorité, donc du retour sur soi. Animée par la volonté ou le désir, la main qui touche est donc un organe privilégié de la sensibilité, qui accueille la sensation et s'y confond, à « l'écoute » du milieu qu'elle reçoit et dont elle prend la mesure. René Passeron dit même d'elle qu'« elle est douée de flair tactile $^{48}$. » Elle est le geste d'ouverture qui permet ceux d'analyse, d'interprétation et de réaction qui viennent ensuite. 
Dans Phénoménologie de la perception, on sent confusément l'impossible synchronisation du sujet et de la sensation, due à «l'épaisseur d'un acquis originaire qui empêche mon expérience d'être claire pour elle-même ${ }^{49}$. » De quel « acquis originaire » peut-il s'agir et en quoi cela influence-t-il radicalement notre vision des modes de connaissance ? On en revient aux trois temps de la main évoqués tout à l'heure par Derrida. L'« effort » de réflexion accompli par le sujet qui perçoit le monde est proposé par Merleau-Ponty comme " vérification » d'une perception originaire, «fonds irréfléchi », une expérience «non thétique, préobjective et préconsciente» relevant de «l'adhésion globale au monde ».

Nous concevons pour notre part que la matière et la forme de la connaissance sont des résultats de l'analyse. Je pose une matière de la connaissance, lorsque, rompant avec la foi originaire de la perception [je souligne], j'adopte à son égard une attitude critique et je me demande "ce que je vois vraiment". La tâche d'une réflexion radicale, c'est-à-dire de celle qui veut se comprendre elle-même, consiste, d'une manière paradoxale, à retrouver l'expérience irréfléchie du monde, pour replacer en elle l'attitude de vérification et les opérations réflexives, et pour faire apparaître la réflexion comme une des possibilités de mon être. Qu'avons-nous donc au commencement? Non pas un multiple donné avec une aperception synthétique qui le parcourt et le traverse de part en part, mais un certain champ perceptif sur fond de monde ${ }^{50}$.

La remarque est fondamentale. Elle rejoint Marcel Mauss, Bernard Stiegler, Gilbert Simondon et Gaston Bachelard sur la question des origines, donc de la technique, par rapport à la pensée magique du monde. On a d'abord une "foi originaire de la perception ", qui relève d'une harmonie originelle avec le monde, formulée par Simondon comme "l'unité magique primitive». L'univers est éprouvé comme un milieu auquel l'homme se trouve lié sans distinction objective. Autrement dit, «le mode primitif d'existence de l'homme dans le monde correspond à une union primitive, avant tout dédoublement, de la subjectivité et de l'objectivité ${ }^{51}$. » Le monde est reçu par une sorte de perception pure, ou d'« expérience irréfléchie » (qui correspondrait, si l'on veut, au premier temps de la main, celui de la réceptivité, qu'évoquait Derrida). On retrouve chez M. Merleau-Ponty et G. Simondon l'idée d'un "fond du monde", structure réticulaire, qui se scinde ensuite pour donner la technique et la religion. L'homme prend de la distance avec le monde, interposant des outils et des croyances qui n'auront pourtant de cesse de retrouver l'unité originelle. Interposant aussi une analyse, ou une " attitude critique ", une " opération réflexive ", qui est bien le signe de notre extériorité au monde et à nous-mêmes.

Dans de telles conditions, comment alors «retrouver l'expérience irréfléchie du monde »? Bachelard nous en donne peut-être quelques moyens, lorsqu'il établit « une thèse qui affirme le caractère primitif, le caractère psychiquement fondamental de l'imagination créatrice». Il suggère que «le psychisme humain se formule primitivement en images ${ }^{52} »$, avant de devenir des idées. D'où le concept d'«imagination matérielle» (par opposition ou complémentarité à l'« imagination formelle ») qui, à partir des éléments - la terre, le feu... - ou de qualités matérielles comme le dur ou le mou, donnent lieu à ses fameuses "rêveries de la matière ». Ainsi ces «forces imaginantes creusent le fond de l'être»-ou le fond du monde - et les « images directes de la matière » sont rêvées, travaillées à l'écart des formes.

La vue les nomme mais la main les connaît. Une joie dynamique les manie, les pétrit, les allège. Ces images de la matière, on les rêve substantiellement, intimement, en écartant les formes ${ }^{53}$. 

proposée par B. Stiegler d'une « tekhnè qui invente l'homme et non l'homme qui invente la technique ${ }^{54}$ ». La mémoire - ou « l'épigenèse »- naît dans la matière qui se réfléchit sous formes d'images matérielles en l'homme.

71 Bachelard revendique en outre la "puissance individualisante de la matière », qui s'exprime au fond de nous sous forme d'images archétypales, peut-être nées au temps où les hommes ne mettaient pas de distance entre un monde réticulaire et eux.

N'y a-t-il pas une individualité en profondeur qui fait que la matière, en ses plus petites parcelles, est toujours une totalité ? Méditée dans sa perspective de profondeur, une matière est précisément le principe qui peut se désintéresser des formes. Elle n'est pas le simple déficit d'une activité formelle. Elle reste elle-même en dépit de toute déformation, de tout morcellement ${ }^{55}$.

Matière étendue, premier support de l'imagination matérielle de l'homme, qui, avant tout projet formel, se livre à une exploration des matières en fonction de leurs qualités plastiques, de leur résistance ou de leur souplesse, suivant, peut-être, la fantaisie de son désir, de ses pulsions, ou de sa perception. Ou suivant le principe de nécessité, ou de manque, qui l'a amené à regarder les choses sous un autre angle, celui de sa survie. C'est ici que la main qui touche cède le pas aux mains qui prennent.

\section{Les mains qui prennent}

Atant la main qui touche est celle, indéterminée ou curieuse, qui explore, tâtonne et interroge, autant celles qui prennent marquent la volonté. Il n'est pas dit qu'elles n'explorent plus, mais dans le fait même de la prise en main, s'exprime une intentionnalité, un mode d'action qui engage celui qui se saisit à faire quelque chose. Jean Brun l'exprime en ces termes : «La main qui prend vise à comprendre, la main qui touche espère parvenir à connaître ${ }^{56}$. " La main in-formée en effet revient toujours à soi vide et nue, elle n'a pas cherché à retenir (au sens propre) l'objet de son exploration, ni à le trans-former. Elle reste détachée mais retient la forme qui s'est imprimée (et peutêtre déjà déformée) dans la mémoire ; elle est un organe de mesure et de connaissance, de pressions et d'impressions, de souvenir et d'émotions. Elle s'organise dans l'absence qui suit le toucher. Ce n'est d'ailleurs pas un hasard si elle a donné lieu aux unités de mesure primitives : le pouce, le doigt, l'empan, la poignée, la pincée etc. Elle mesure, elle est instrument de mesure.

74 Les mains qui prennent sont à la fois plus puissantes et moins agiles que celles qui touchent, car, comme on l'a déjà vu, elles renoncent aux subtilités du toucher pour se refermer comme des pinces sur ce qu'elles tiennent. On prend pour porter, ou pour porter à (la bouche, la vue, l'oreille etc.). On prend un peu de terre pour la modeler, un morceau de pain pour le manger, une arme pour agresser ou se défendre, etc. La prise en main introduit une suite ou une rupture par rapport au «simple » toucher, une geste déterminé qui fait passer la sensation derrière l'action.

75 Elles s'attachent à ce qu'elles font, elles se referment sur ce qu'elles contiennent et s'apprêtent à agir; "grâce à la prise, la main tient, rompt ou joint, beaucoup plus qu'elle ne forme, elle déforme pour reformer et façonner ${ }^{57}$. " On a tendance à oublier que les mains articulées, prolongées par l'outil, tendent d'abord à arracher la matière à sa forme - ou à son absence de forme - initiale par un geste de déformation. Le mineur casse la roche pour extraire le métal du forgeron, le potier prélève l'argile du sol avant 
de la battre pour lui redonner une unité, le bûcheron coupe les arbres pour le menuisier ou le charpentier, etc. Double mouvement d'un travail des matières qui commence par une destruction, une déformation ou une soustraction avant d'obtenir par intégration à un ensemble d'opérations techniques de remodelage une nouvelle unité fabriquée. C'est à ce point de rencontre entre l'activité et la main que se situent les outils, les instruments, les appareils.

Henri Focillon emprunte au langage des peintres le terme de " touche », correspondant au moment où « l'outil éveille la forme dans la matière ${ }^{58}$. » Elle est d'abord un contact, un point dans l'espace et le temps entre une matière inerte et une action qui lui donnera forme. Un mouvement du corps qui se caractérise d'abord par son action et ensuite par son mode d'action, qui est aussi l'identité de celui qui pose cette touche. En cela, le geste est propre à chacun, donc unique et identifiable.

La touche est structure. Elle superpose à elle de l'être ou de l'objet la sienne propre,

sa forme, qui n'est pas seulement valeur et couleur, mais (même dans des

proportions infimes) poids, densité, mouvement ${ }^{59}$.

On pense au bâton des aveugles qui touche les choses et les « donne à voir » à celui qui n'a d'autre sens pour appréhender l'espace. L'outil pris en main et manipulé de telle ou telle façon devient ainsi l'organe artificiel qui pose en matière le regard ou l'identité de celui qui le tient. Avec le langage du toucher, on compose celui de la vue. La touche - du peintre, du sculpteur, de l'artisan, du chirurgien même - est un geste technique qui fait aussi passer par l'outil tout le poids du corps, l'art et la manière (le toucher) de celui qui le tient. L'art et la manière d'intervenir sur l'ordre auquel il est soumis, d'inventer à partir de ce monde régi par des lois naturelles un autre univers qui sort de lui. L'outil lui-même, qui est un objet inerte, prend vie dans la main qui le tient: il se "fait " à l'usure, s'harmonise avec celui qui l'utilise, prend la forme de ses gestes. Un artisan rechigne à prêter ses outils, qui ne sont jamais meilleurs que quand ils ont "pris le pli » de sa main. Un outil en bois (ou dont le manche est en bois) s'y prête mieux ou plus vite que tout autre, car il est organique, souple et s'adapte vite au corps qui le manipule, mais c'est vrai aussi pour les outils métalliques, qui s'« échauffent » petit à petit à la chaleur du corps. À cet égard, l'outil neuf est comme un nouveau compagnon de travail encore étranger avec lequel on tisse petit à petit une complicité. Les mains qui prennent touchent aussi ce qu'elles prennent, leur impriment une facture qui passe à travers les outils dans la matière façonnée.

Finalement, qu'elles prennent ou qu'elles touchent, elles portent surtout en elles le temps d'une gestation, qui est la marque du travail. C'est bien le sens premier de "geste », gerere, qui signifie d'abord "porter sur soi " avant de vouloir dire «faire, exécuter ». Et le dérivé gestus est "le mode de l'action à accomplir ", "l'attitude du corps $^{60} »$, l'art et la manière donc. Un geste est donc avant tout expressif, il ne peut être qu'expressif dans la mesure où il est effectué par un être vivant, un corps unique et singulier qui lui donne sa forme et sa fonction. Ensuite seulement est-il plastique, technique et productif. Un geste des mains, c'est donc un mouvement de tout le corps et de l'esprit qui cherchent une forme à travers elles et les outils qu'elles manipulent.

On a vu que la main de l'homme en tant que dispositif ostéo-musculaire n'a rien d'extraordinaire par rapport à celle des primates et qu'elle permet de réaliser les mêmes opérations de base, comme la préhension, la manipulation, le pétrissage, la percussion ou l'épluchage. Ce n'est donc pas dans l'appareillage moteur que se crée la différence entre leurs mains et les nôtres : 
La main est humaine par ce qui s'en détache et non par ce qu'elle est [...] La valeur humaine du geste n'est donc pas dans la main, dont la condition suffisante est qu'elle soit libre pendant la marche, mais précisément dans la marche verticale et dans ses conséquences paléontologiques sur le développement de l'appareil cérébral ${ }^{61}$. autour d'elle des éléments du monde pour compenser son manque de technicité. Une main appareillée, prothétique, qui se saisit d'abord des « outils » que lui offre la nature (sans chercher à les transformer) pour effectuer les opérations de percussion strictement nécessaires, puis, et c'est là l'incroyable révolution, qui se met à agir sur ces outils naturels pour les perfectionner. La main ne frappe plus seulement avec une pierre, elle redouble son action et frappe cette pierre elle-même avec une autre pierre jusqu'à obtenir un outil à proprement parler, c'est-à-dire une pierre qui n'existait pas auparavant, un objet fabriqué, manu-facturé, plus tranchant et mieux adapté aux opérations qu'il doit réaliser. Premier signe d'une mètis au service de la techné, première création démiurgique au sens originel du terme latin crescere, «faire pousser, faire grandir, produire ». Nos mains « font pousser » la matière silex, par exemple, déjà tranchante en soi, la rendant encore plus acérée; elles perçoivent les qualités des matériaux et les poussent plus loin. Besoin de perfectibilité qui semble aussi vieux que nous. Nos mains fabriquent de la valeur ajoutée à la nature.

La différenciation se fait donc d'une part dans l'enrichissement de la sensibilité tactile et surtout dans l'évolution des processus opératoires liés à l'extériorisation de nos gestes techniques. Dès qu'apparaissent les premiers silex taillés, les opérations techniques émigrent dans les outils: "La main cesse d'être outil pour devenir moteur ${ }^{62}$.» A. Leroi-Gourhan décompose les différentes étapes de notre motricité manuelle, de l'action manipulatrice à la motricité indirecte, dans laquelle la main "déclenche un processus programmé dans les machines automatiques », en passant par la motricité directe, où l'outil manuel était « inséparable du geste moteur ».

Notre main quant à elle reste un organe archaïque et très peu spécialisé, capable pourtant d'opérations manipulatrices complexes comme la rotation et la translation. Selon l'évolution biologique qui se caractérise par des «libérations» successives (des mains, de la face, du cerveau), la main se libère aussi progressivement de sa motricité, qui passe dans des machines manuelles avant d'être relayée par une motricité animale ou automotrice dans les machines agissant grâce au vent, à l'eau, puis, progrès décisif, grâce à la pression de la vapeur et enfin à l'électricité, garantissant une force motrice illimitée.

Ainsi, le geste technique s'inscrit paradoxalement dans une logique biologique de libération qui le conduit pourtant à une dépendance unique dans le règne du vivant à ses prothèses, posées hors de lui et soumises elles-mêmes à une évolution qui se rejoue à chaque génération qui s'en saisit. Ses stratégies d'adaptation, qui ont fait de lui l'animal technicien le plus puissant de tous, sont donc autant de chances et de pièges, qui lui offrent tour à tour la possibilité de créer ses propres conditions de vie, de jouir d'une liberté inégalée et inégalable par rapport au milieu dans lequel il évolue, et qui risquent à tout moment de l'en déconnecter. Gageons cependant que l'être " ouvert » saura une fois de plus trouver le moyen de préserver sa démiurgie sans pour autant devenir un intrus dans son propre monde. 


\section{BIBLIOGRAPHIE}

Bachelard Gaston, L'eau et les rêves, Paris, Librairie José Corti, 1942.

Bachelard Gaston, La terre ou les rêveries de la volonté, Paris, Librairie José Corti, 1948.

Brun Jean, La main et l'esprit, Genève, Labor et Fides, 1986.

Derrida Jacques, Le toucher, Jean-Luc Nancy, Paris, Galilée, 2000.

Dictionnaire étymologique du français, Paris, Dictionnaire le Robert, 1992.

Focillon Henri, Vie des formes, Paris, PUF, 1996.

Guérin Michel, Philosophie du geste, Arles, Actes Sud, 1995.

Huyghe Pierre-Damien, Art et Industrie, Paris, Circé, 1999.

Huyghe Pierre-Damien, Le différend esthétique, Paris, Circé, 2004.

Latour Bruno, L'espoir de Pandore. Pour une version réaliste de l'activité scientifique, Didier Gille (trad.), Paris, La Découverte / Poche, 2007.

Leroi-Gourhan André, Le geste et la parole, tome II : La mémoire et les rythmes, Paris, Albin Michel, 1965.

Mauss Marcel, « Les techniques du corps », in Sociologie et anthropologie, Paris, PUF Quadrige, 2004.

Merleau-Ponty Maurice, Phénoménologie de la perception, Paris, Gallimard, 1993.

Nancy Jean-Luc, « De l'âme », Corpus, Paris, Métailié, 2000.

Nancy Jean-Luc, L'Intrus, Paris, Galilée, 2000.

Passeron René, L'œuvre picturale et les fonctions de l'apparence, Paris, Vrin, 1994.

Séris Jean-Pierre, La technique, Paris, PUF, 1994.

Simondon Gilbert, Du mode d'existence des objets techniques, Paris, Aubier, 1958, 1989.

Stiegler Bernard, La technique et le temps I, La faute d'Epiméthée, Paris, Galilée, 1994.

Vercors, Les animaux dénaturés, Paris, Livre de Poche, 1952.

\section{NOTES}

1. Jean-Luc Nancy, De l'âme, conférence prononcée le 8 avril 1994 lors du colloque intitulé «Le poids du corps » et retranscrite dans Corpus, Paris, Métailié, 2000, p. 108.

2. Ibid., p. 113.

3. Gaston Bachelard, L'eau et les rêves, Paris, Librairie José Corti, 1942, p. 145.

4. Pierre-Damien Huyghe, Le différend esthétique, Paris, Circé, 2004, p. 12.

5. Ibid., p. 13.

6. Gilbert Simondon, Du mode d'existence des objets techniques, Paris, Aubier, 1958, 1989, p. 74.

7. Ibid., p. 73.

8. Gaston Bachelard, La terre ou les rêveries de la volonté, Paris, Librairie José Corti, 1948, p. 3.

9. Bernard Stiegler, La technique et le temps I, La faute d'Epiméthée, Paris, Galilée, 1994, p. 63.

10. Marcel Mauss, "Les techniques du corps ", in Sociologie et anthropologie, Paris, PUF Quadrige, 1950, 2004, p. 372. 
11. Ibid., p. 374.

12. Pierre-Damien Huyghe, Art et Industrie, Paris, Circé, 1999, p. 58.

13. On notera la remarque qui suit entre parenthèses : ( comme on dit, croyant du reste savoir ce que c'est qu'un “objet technique” »), Jean-Luc Nancy, Corpus, p. 79. Jean-Luc Nancy n'a peutêtre pas lu l'ouvrage de Gilbert Simondon, qui nous éclaire un tant soit peu sur la notion d'« objet technique $»$.

14. Ibid., p. 78.

15. Ibid., p. 85.

16. Jean-Pierre Séris, La technique, Paris, PUF, 1994, p. 70.

17. Un corps « dénaturé » comme l'homme peut être un « animal dénaturé » au sens où l'entend Vercors: "L'animal fait un avec la nature. L'homme fait deux. Pour passer de l'inconscience passive à la conscience interrogative, il a fallu ce schisme, ce divorce, il a fallu cet arrachement. N'est-ce point la frontière justement? Animal avant l'arrachement, homme après lui ? Des animaux dénaturés, voilà ce que nous sommes. " Vercors, Les animaux dénaturés, Paris, Livre de Poche, 1952, p. 195.

18. Bruno Latour, L'espoir de Pandore. Pour une version réaliste de l'activité scientifique, Cambridge, 1999, trad. de l'anglais Didier Gille, Paris, La Découverte / Poche, 2007, p. 189.

19. Ibid., p. 227.

20. Paru dans La Revue d'Anthropologie des Connaissances qui aborde le geste technique, Paris, Erès, 2002, p. 283.

21. Éric Gallais et Ali Ahmed Saïd, «Les coordonnées culturelles du geste», La Revue d'Anthropologie des Connaissances, p. 285.

22. Ibid.

23. Michel Guérin, Philosophie du geste, Arles, Actes Sud, 1995, p. 29.

24. Ibid., p. 16.

25. Jean-Luc Nancy, « De l'âme », in Corpus, p. 107.

26. Maurice Merleau-Ponty, Phénoménologie de la perception, p. 268.

27. Ibid., p. 127.

28. Jean-Luc Nancy, « De l'âme » in Corpus, p. 122.

29. Ibid., p. 122.

30. Ibid., p. 127.

31. Jacques Derrida, Le toucher, Jean-Luc Nancy, Paris, Galilée, 2000, p. 284.

32. Ibid., p. 102.

33. Édition datée du $1^{\mathrm{er}}$ février 2006, p. 24.

34. Ibid.

35. Jean-Luc Nancy, L'Intrus, Paris, Galilée, 2000, p. 12.

36. Ibid., p. 45. (Ce sont les derniers mots du livre.)

37. Ibid., p. 44.

38. Jacques Derrida, Le toucher, Jean-luc Nancy, p. 113.

39. Ibid., p. 252.

40. Jean-Luc Nancy, Corpus, p. 47.

41. Jean Brun, La main et l'esprit, Genève, Labor et Fides, 1986, p. 110. On se demande toutefois si la perception, le « ressenti » que semble minimiser l'auteur, n'est pas indissociable du "sentir ", comme les deux faces d'une même médaille.

42. Ibid., p. 112.

43. Ibid., p. 109.

44. Jacques Derrida, Le toucher, Jean-Luc Nancy, p. 174.

45. Ibid., p. 174.

46. Ibid., p. 176. 
47. Et surtout, dépassons les préjugés qui relèvent de la supériorité des hommes, qui ont des mains, sur les animaux, qui n'en ont pas, ou qui ne savent pas aussi bien s'en servir.

48. René Passeron, L'œuvre picturale et les fonctions de l'apparence, Paris, Vrin, 1962, 1994, p. 69.

49. Maurice Merleau-Ponty, Phénoménologie de la perception, Paris, Gallimard, 1979, 1993, p. 261.

50. Ibid., p. 288.

51. Gilbert Simondon, Du mode d'existence des objets techniques, p. 166.

52. Gaston Bachelard, La terre et les rêveries de la volonté, p. 5.

53. Gaston Bachelard, L'eau et les rêves, p. 2.

54. Bernard Stiegler, La technique et le temps I, p. 152.

55. Gaston Bachelard, L'eau et les rêves, p. 3.

56. Jean Brun, La main et l'esprit, p. 113.

57. Ibid., p. 138.

58. Henri Focillon, Vie des formes, Paris, PUF, 1996, p. 63.

59. Ibid., p. 64.

60. Dictionnaire étymologique du français, Paris, Dictionnaire le Robert, 1992.

61. André Leroi-Gourhan, Le geste et la parole, tome II : La mémoire et les rythmes, Paris, Albin Michel, 1965, p. 41.

62. Ibid., p. 41.

\section{RÉSUMÉS}

Le geste technique peut être pensé comme l'articulation d'une défaillance à une ouverture. La défaillance d'un corps "sans qualité " et l'ouverture qui, avant même de s'inscrire dans une extériorisation par l'outil, passe par une plasticité ou, pour parler comme Merleau-Ponty, par une « saisie des coexistences » propre au toucher.

L'efficacité de la technique, la maîtrise du geste puis de l'environnement, l'organisation prothétique du corps repose avant tout sur une plasticité qui permet à l'homme de sortir du strict fonctionnement biologique. C'est en cela qu'il ex-iste, qu'il se tient en dehors des limites de son corps, qui, mystère des origines, est devenu progressivement un organe de perception capable de se réfléchir dans la matière jusqu'à la transformer, jusqu'à modeler une technicité organisée de manière unique au sein du vivant: l'« écotechnie des corps ». C'est en ces termes que Jean-Luc Nancy désigne les corps articulés par la technique, intégrés dans un système d'appareillage qui fait du corps humain un corps connecté, impensable sans ses outils. Tout geste est-il donc d'abord un geste technique?

Cet article propose une analyse ontologique du geste technique à partir de considérations paléontologiques et philosophiques - notamment le concept d'imagination matérielle développé par Gaston Bachelard - avec pour point de départ le sens du toucher comme origine de la prothéticité de l'être humain, et un questionnement sur les limites de ce corps « dressé », dont la maîtrise, ainsi que celle de son milieu, est un enjeu de pouvoir et d'existence, mais aussi un risque. 
INDEX

Mots-clés : appareillage, apprentissage, artisan, corps, dressage, efficacité, geste, machine, main, maitrise, matière, moyen, norme, outil, technè, technique, toucher

\section{AUTEUR}

\section{PATRICIA RIBAULT}

Docteur en arts, esthétique et sciences de l'art de l'université de Paris I Panthéon-Sorbonne, responsable de la recherche à l'École supérieure d'art et de design de Reims, chargée de cours au Conservatoire national des arts et métiers, enseignante à l'École nationale supérieure des BeauxArts de Paris ciotola@free.fr 